\title{
GAMES IN CODE FORM-INTRODUCING A NEW GAMES REPRESENTATION
}

\author{
Prof. Dr. Manuel Alberto M. Ferreira ${ }^{1}$, Prof. Dr. Maria Cristina Peixoto Matos ${ }^{2}$, \\ Prof. Dr. José António Filipe ${ }^{1}$ \\ ${ }^{1}$ Instituto Universitário de Lisboa (ISCTE - IUL), BRU - IUL, Lisboa, \\ ${ }^{2}$ Instituto Politécnico de Viseu - ESTV, Viseu (PORTUGAL) \\ E-mails: manuel.ferreira@iscte.pt, cristina.peixoto@math.estv.ipv.pt, Jose.Filipe@iscte.pt
}

DOI: $10.7813 / 2075-4124.2014 / 6-1 / A .7$

Received: 10 June, 2013

Accepted: 25 Dec, 2013

\begin{abstract}
The objective of this paper is to present a new representation for games with more than two players. This formalization concentrates the consequences of the various strategies without the suppression of the extensive form details. In other words, this representation supplies simultaneously the whole information that the extensive and normal forms establish separately. Some games are presented in order to illustrate these ideas.
\end{abstract}

Key words. Games theory, extensive form, normal form, code form

MSC 2010:91A80

\section{INTRODUCTION}

The idea of a new representation for games was suggested by the analysis of a game with three players in the normal form. It occurred that a representation that gives a global image of the game would be the ideal one. As a consequence, it was made the tentative of representing a game that included both the information's of the extensive form and of the normal form.

The code form representation was then imagined and it was checked that this representation has great advantages when the number of players is greater or equal than three, when the game is on imperfect information and mainly when the game is sequential.

Before presenting the code form, the already commonly used games representations are remembered.

\section{GAMES REPRESENTATION}

The games are usually represented in two ways:

- The normal form,

- The extensive form

\section{Definition 2.1 (Normal form)}

A game with $n$ players is any list $G=\left(S_{1}, S_{2}, \ldots, S_{n} ; u_{1}, u_{2}, \ldots, u_{n}\right)$, where, for each $i \in N=\{1,2, \ldots, n\}, S_{i}$ is the set of the whole available strategies for player $i$, and $u_{i}=S_{1} \times S_{2} \times \ldots, \times S_{n} \rightarrow \mathbb{R}$ is the utility function for player i..

\section{Observation:}

- For each player its utility depends not only on its strategy but also on the strategies chosen for the other players. But $u_{i}$ is a strategy such that player $i$, when choosing its strategy has the objective of maximizing the $u_{i}$ expected value. It is also assumed that there are $n$ players and that the set of available strategies for each player $i$ is $S_{i}$, and that each $i$ has, as objective, to maximize the $u_{i}$ expected value.

- The extensive form contains the whole information about the game, defining the whole moves and when they occur, what is known for each player when it plays: which moves are available for it, and to where each move leads. 


\section{Definition 2.2}

A tree is a set of nodes and arcs that connect the nodes in the following way:

-For each node there is at least one arc,

-For any two nodes, there is one only path that connect these two nodes.

\section{Definition 2.3}

A game in the extensive form consists in a set of players, a tree, an assignment to each node of the tree, with the exception of the terminal ones, for each player, an informative partition, and payoffs for each player in every terminal node.

\section{Observation:}

- The set of players includes the agents that belong to the game. But, in a lot of games there is place for gambling. That is, there is gambling whenever there is uncertainty on any relevant fact. To represent these possibilities it is added a fictitious player: the nature. There are no payoffs for the nature in the terminal nodes. And when an arc is assigned to the nature it is specified, above that arc, the corresponding probability distribution.

\section{Definition 2.4}

An information set for player $i$ is a collection of nodes $\left\{m_{1}, m_{2}, \ldots, m_{k}\right\}$, such that the player $i$ move along these nodes and there are possible moves at each one of these nodes.

\section{Definition 2.5}

An informative division is an assignment of each node of the tree to an information set.

\section{Definition 2.6}

A strategy for a player is a complete action plan, that states which action it will take in each information set at which it moves.

Then the code form will be introduced in the next section. It will be made step by step, standing on games with different characteristics and comparing it with representations of those games in the forms normal and extensive.

\section{PERFECT INFORMATION}

Consider a game with three players. Each player has two available strategies: " $D$ ". and " $E$ ". If no player adopts the strategy " $D$ ", the results vector will be $(0,0,0)$. If exactly one of them adopts the strategy " $D$ ", that player obtains the payoff 1 while every player that adopts the strategy " $E$ " obtains a payoff of $-\frac{1}{2}$. For instance, if only player 1adopts the strategy " $D$ ", the results vector is $\left(1,-\frac{1}{2},-\frac{1}{2}\right)$. If exactly two players, for instance player 1 and player 2 , adopt " $D$ " the results vector is $\left(\frac{1}{2}, \frac{1}{2},-1\right)$. Finally, if the whole players adopt " $D$ "the results vector is $(0,0,0)$.

\subsection{Extensive Form}

In Fig. 1 is presented the former game in the extensive form.

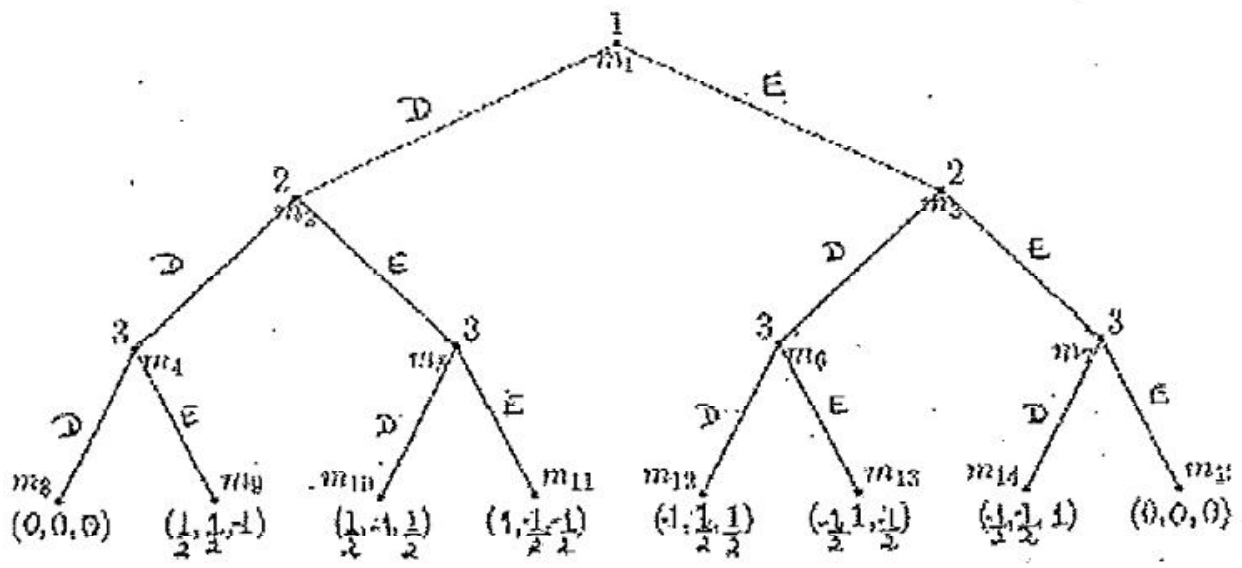

Fig. 1. Perfect Information, three players - Extensive form 
The nodes labelled $m_{1}, m_{2}, \ldots, m_{15}$ represent the various states of the game. The node codified with the number $1, m_{1}$, indicates that the player that makes the first move is the player 1 . The two arcs that depart from this node indicate that this player has two possible choices here: to opt either for the strategy " $D$ "or by the strategy " $E$ ".. Both nodes lead to nodes codified with the number 2, $m_{2}$ and $m_{3}$, what means that after the move of player 1 is the time for player 2 move. There are two arcs departing from each one of those nodes. This means that at each one of those nodes - one resulting from the option of player 1 by " $D$ "and the other from the option of player 1 by " $E$ ". - player 2 has two options: choose " $D$ " or choose " $E$ ".. Each one of these actions leads to four nodes, $m_{4}, m_{5}, m_{6}$ and $m_{7}$ - result from the options of players 1 and $2-$ codified by 3 what means that is the time for this player moves. Each one of those nodes leads to a terminal node. The three elements ordered sequences in the terminal nodes $m_{8}, m_{9}, \ldots, m_{15}$, indicate the payoffs of the game.

To find the game solution it is used the backward induction method. At every one of its terminal nodes, player 3 has to take a decision in order to maximize its payoff. So it is concluded that its strategy is $s_{3}^{*}=$ ("D", "D", "D", "D").

Going back to player 2, with the same criterion as player 3 , the strategy that it chooses is $s_{2}^{*}=\left(" D^{\prime}, " D^{\prime \prime}\right)$.

Finally, going back to player 1 , its optimal decision is $s_{1}^{*}=(" D ")$.

So, through the backward induction method, only one strategy profile results - $\left(s_{1}^{*}, s_{2}^{*}, s_{3}^{*}\right)$ - from where it follows that the game solution corresponds to the payoff $(0,0,0)$.

\subsection{Normal Form}

As referred before, the normal form of a game is a resume of the game itself that pays attention only to the strategies and to the payoffs so resulting. In consequence, a lot of the game information is lost as it is possible to check analysing Fig. 2, which shows the game in normal form.

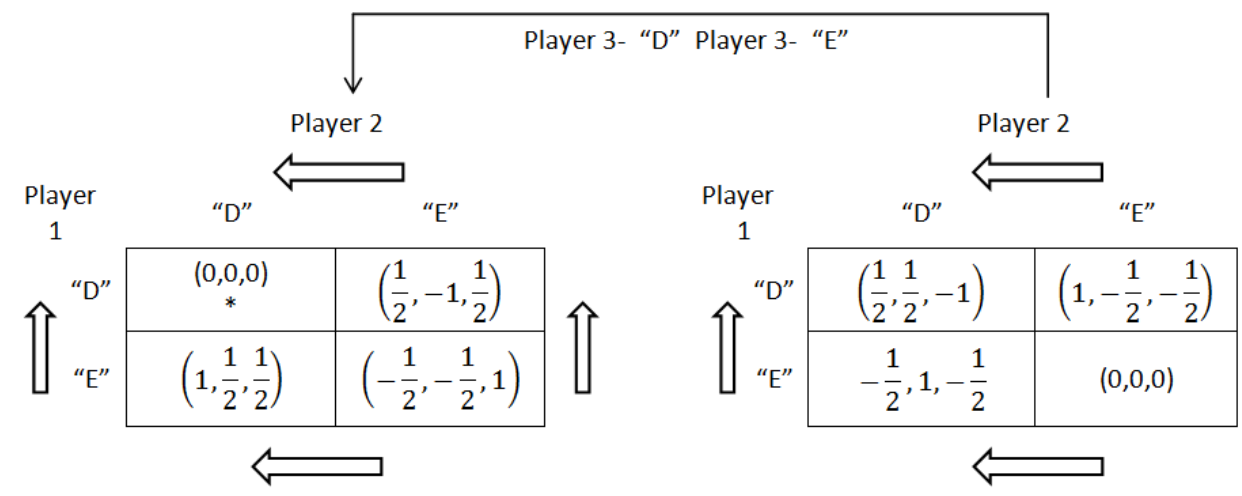

Fig. 2. Perfect Information, three players - Normal form

In Fig. 2, the left arrow that points up indicates that, when players 2 and 3 opt both by the strategy " $D$ ", the player 1 prefers to choose " $D$ " getting a payoff of 0 instead of choosing " $E$ " and to get a payoff of -1 . In a similar way, the arrow located below that points to the left side indicates that the player 2 prefers to choose " $D$ " and to get a payoff of $\frac{1}{2}$ instead of choosing " $E$ " and to get a payoff of $-\frac{1}{2}$ when the player 1 opts by the strategy " $E$ " and player 3 by the strategy " $D$ ". Reasoning similarly the whole other possibilities are analysed. There is only one case at which the arrows point in the same direction: the players 1,2 and 3 prefer the strategy " $D$ " that guarantees a better payoff. This situation is indicated by $a *$.

Analysing the arrows diagram it is concluded that the only equilibrium occurs when the whole players adopt the strategy " $D$ ", a result that was obtained through the induction method.

\subsection{Code Form}

The idea of a code form game representation stands on the presupposition of a linear reading of the game. It is built a table with the whole information of the game. How to organize the information?

In Fig. 3 it is presented the former described game code form. 


\begin{tabular}{|c|c|c|c|c|}
\hline 1 & $\left(1,{ }^{\prime \prime} \mathrm{D}^{\prime \prime}\right)$ & & & \\
\hline 2 & & $\left(2,{ }^{\prime \prime} \mathrm{D}^{\prime \prime}\right)$ & & \\
\hline 3 & & & $\left(3,{ }^{\prime \prime} D^{\prime \prime}\right)$ & $(0,0,0)$ \\
\hline 3 & & & $\left(3,{ }^{\prime \prime} E^{\prime \prime}\right)$ & $\left.\left(\frac{1}{2}, \frac{1}{2},-1\right)\right)$ \\
\hline 2 & & $\left(2,{ }^{\prime \prime} \mathrm{E}^{\prime \prime}\right)$ & & \\
\hline 3 & & & $\left(3,{ }^{\prime \prime} D^{\prime \prime}\right)$ & $\left.\left(\frac{1}{2},-1, \frac{1}{2}\right)\right)$ \\
\hline 3 & & & $\left(3,{ }^{\prime \prime} E^{\prime \prime}\right)$ & $\left.\left(1,-\frac{1}{2},-\frac{1}{2}\right)\right)$ \\
\hline 1 & $\left(1,{ }^{\prime \prime} E^{\prime \prime}\right)$ & & & \\
\hline 2 & & $\left(2, " D^{\prime \prime}\right)$ & & \\
\hline 3 & & & $\left(3,{ }^{\prime \prime} D^{\prime \prime}\right)$ & $\left.\left(-1, \frac{1}{2}, \frac{1}{2}\right)\right)$ \\
\hline 3 & & & $\left(3,{ }^{\prime \prime} \mathrm{E}^{\prime \prime}\right)$ & $\left.\left(-\frac{1}{2}, 1,-\frac{1}{2}\right)\right)$ \\
\hline 2 & & $\left(2,{ }^{\prime \prime} E^{\prime \prime}\right)$ & & \\
\hline 3 & & & $\left(3,{ }^{\prime \prime} D^{\prime \prime}\right)$ & $\left.\left(-\frac{1}{2},-\frac{1}{2}, 1\right)\right)$ \\
\hline 3 & & & $\left(3,{ }^{\prime \prime} E^{\prime \prime}\right)$ & $(0,0,0)$ \\
\hline
\end{tabular}

Fig. 3. Perfect Information, three players - Code form

Reading from the left to the right, the first column in the table indicates the number of the move. If the players play simultaneously it is filled with the code " $S$ ". The following columns refer to each one of the players and the last column indicates the payoffs vector in accordance with the strategies chosen by the various players. Each player column refers the number of the player and the strategy it chooses. The order of the players is arbitrary when the moves are simultaneous. The fields not filled repeat the information of the former row. The table is built in accordance with the following algorithm:

- Be $m$ the game initial node and suppose that $i$ is the player that has to take a decision.

- Be $s_{i} \in S_{i}$ an action that links the game to a new node. The $2^{\text {nd }}$ column is filled with $\left(i, s_{i}\right)$. Call $m^{1}$ the following node.

- If $m^{1}$ is a terminal node the last column is filled with the associated payoff and then one is back to the initial node till the player $i$ decision possibilities are exhausted.

- If $m^{1}$ is not a terminal node, one moves to the column immediately at the right of the column and to the row immediately below the player $i$ played row and the process is repeated for the player that decides in the node $m^{1}$. When the moves are simultaneous there is no row change.

The game solution, in code form, is determined analysing the various players' different payoffs: The process begins by selecting the optimal payoff of a player in each one of its information sets. In the end of this selection it is begun an identical process for the player that played immediately before this player but considering now only the before selected payoffs that begin to its information sets. The process goes on till the whole payoffs associated to the player that moved in first place are analysed.

\section{Detailing:}

- In the present game there are 3 players and the last player to move was player 3 for which there are 4 information sets, each one of them with 2 associated payoffs:

$$
\begin{gathered}
\left\{(0,0,0),\left(\frac{1}{2}, \frac{1}{2},-1\right)\right\},\left\{\left(\frac{1}{2},-1, \frac{1}{2}\right),\left(1,-\frac{1}{2},-\frac{1}{2}\right)\right\},\left\{\left(-1, \frac{1}{2}, \frac{1}{2}\right),\left(-\frac{1}{2}, 1,-\frac{1}{2}\right)\right\} \\
\text { and }\left\{\left(-\frac{1}{2},-\frac{1}{2}, 1\right),(0,0,0)\right\} .
\end{gathered}
$$

In the first set the optimal payoff for player 3 is $(0,0,0)$, in the second set is $\left(\frac{1}{2},-1, \frac{1}{2}\right)$, in the third set is $\left(-1, \frac{1}{2}, \frac{1}{2}\right)$ and finally in the fourth set the optimal payoff is $\left(-\frac{1}{2},-\frac{1}{2}, 1\right)$. Ended the analysis referring the player 3 , analyse the payoffs selected referring to the player 2 information sets, since it is this player that plays immediately before the player 3 . With the four payoffs selected for player 3 the two sets associated to player 2 are built:

$$
\left\{(0,0,0),\left(\frac{1}{2},-1, \frac{1}{2}\right)\right\} \text { and }\left\{\left(-1, \frac{1}{2}, \frac{1}{2}\right),\left(-\frac{1}{2},-\frac{1}{2}, 1\right)\right\} \text {. }
$$

The most favourable payoffs for player 3 are exactly $(0,0,0)$ and $\left(-1, \frac{1}{2}, \frac{1}{2}\right)$. To find the equilibrium, it misses to analyse these payoffs from the player 1 point of view. The optimal payoff for player 1 indicates the game solution. This payoff is $(0,0,0)$ that is consequence of the adoption of the strategy " $D$ " by the whole players. This result is confirmed by the arrows diagram and by the induction method former used. 


\subsection{Conclusion}

When the various representations for this game are analysed, it is checked the extensive form and the code form present advantages versus the normal form once those representations allow visualizing the whole game that is the whole information related to the game. But, the code form, when compared with the extensive form, has a simpler lecture and allows, in a linear form, to find the game equilibrium through the payoff vectors.

\section{IMPERFECT INFORMATION}

Now the following game ids considered:

- Three players may choose one of the two options: option "a" and option " $b$ ". Suppose that the player 1 prefers "a" instead of " $b$ ", the player 2 prefers " $b$ " instead of " $a$ " and the player 3 prefers " $a$ " instead of " $b$ ". the player 1 decides first and communicates to the others its decision. With this information the other players make their decision simultaneously. The most voted option is the one chosen.

\subsection{Extensive Form}

In Fig. 4 it is presented the former described game extensive form.

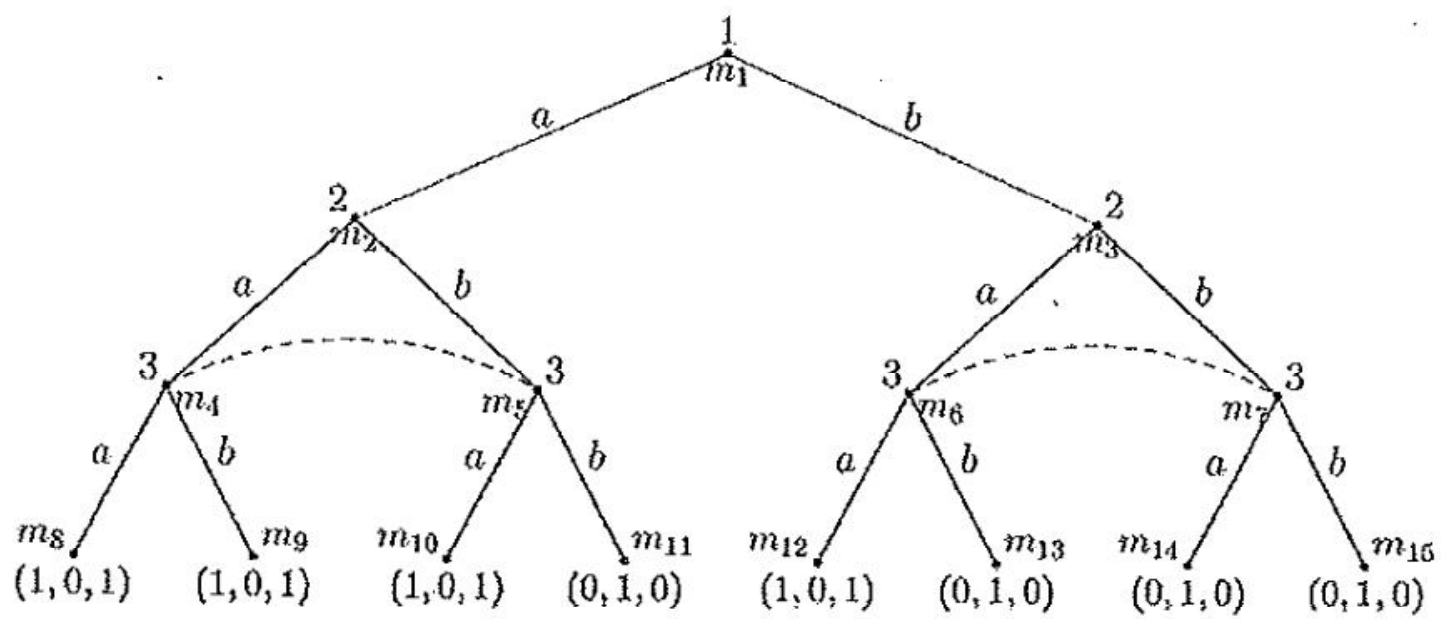

Fig. 4. Imperfect Information, three players - Extensive form

The dotted line that connects the nodes $m_{4}$ and $m_{5}$ indicates that the player 3 does not know the actual state of the game, that is: this player does not know the player 2 option. The nodes $m_{4}$ or $m_{5}$ are not connected to anyone of the nodes $m_{6}$ and $m_{7}$ (nodes at which the player 3 has to play) because the player 3 knows the decision made by player 1 when it is its time to decide. Analogously the dotted line that connects the nodes $m_{6}$ and $m_{7}$ indicates that the player 3 does not know the decision made by player 2. So player 1 has only one information set: $\left\{m_{1}\right\}$; player 2 has two information sets: $\left\{m_{2}\right\}$ and $\left\{m_{3}\right\}$; player 3 has two information sets: $\left\{m_{4}, m_{5}\right\}$ and $\left\{m_{6}, m_{7}\right\}$. Then, when it is time for player 2 to move, it knows that the game is in the state represented by $m_{2}$ or in the state represented by $m_{3}$. When it is time for player 3 to move, it knows that the game is in the state represented by a node of the set $\left\{m_{4}, m_{5}\right\}$ or of the set $\left\{m_{6}, m_{7}\right\}$, but it does not know if this situation corresponds to the nodes $m_{4}, m_{5}, m_{6}$ or $m_{7}$.

To determine this game solution it is not possible to use the backwards induction method once it is an imperfect information game. It may be used the subgames perfect equilibrium. A subgame is a part of the game that, when separated from it, is only by itself a game. This game has three subgames. Itself and the subgames $J_{m_{2}}$ and $J_{m_{3}}$, represented in Fig. 5. 

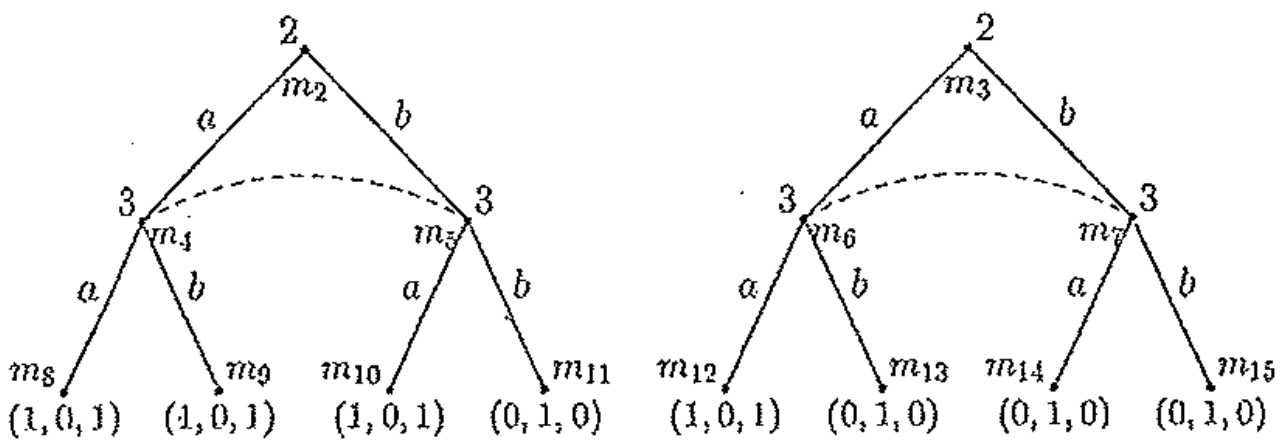

Fig. 5. Subgames - Extensive form

The perfect equilibrium in subgames is determined as follows:

- Look for the equilibrium in each subgame (as the subgames are finite these equilibriums always exist). Then, in the initial game, each subgame is replaced by terminal nodes associating each one of these nodes to the payoff corresponding to the equilibrium determined for each subgame. Finally look for the solution for the obtained game.

Analysing the subgames $J_{m_{2}}$ and $J_{m_{3}}$ it is checked that $(0,1,0)$ is the payoff corresponding to the equilibrium in the subgame $J_{m_{3}}$ and $(1,0,1)$ the payoff corresponding to the equilibrium in the subgame $J_{m_{2}}$. The game resulting (reduced) is represented in Fig. 6.

The reduced game solution is the strategy at which player 1 chooses "a", in the information set $\left\{m_{1}\right\}$. Then the perfect solution in subgames for the initial game is the strategic profile $(a,(a, b), a)$ that corresponds to the payoff $(1,0,1)$

\subsection{Code Form}

In Fig. 6 the game is represented in the code form. When this representation is constructed, it is evident that a code indicating that the game is an imperfect information one must be added and also which player holds imperfect information. Then to column of the player it is linked a code I,that indicates what player has imperfect information, as well as the code of the player for which it holds that kind of information. To allow several possibilities, this field is filled only in the rows that correspond to strategies that imply imperfect information. Whenever the player has perfect information this field is not filled.

Once the code form also allows subgames, its solution, such has it happens with the extensive form, is achieved through prefect equilibrium in subgames.

\begin{tabular}{|c|c|c|c|c|}
\hline 1 & (1,"a") & & & \\
\hline \multirow[t]{4}{*}{$2-s$} & & $(2, " a ", I, 3)$ & $(3, " a ", I, 2)$ & $(1,0,1)$ \\
\hline & & & $(3, " b ", l, 2)$ & $(1,0,1)$ \\
\hline & & $\left(2, " b^{\prime \prime}, I, 3\right)$ & $(3, " a ", 1,2)$ & $(1,0,1)$ \\
\hline & & & $\left(3, "{ }^{\prime \prime}, 1,2\right)$ & $(0,1,0)$ \\
\hline 1 & $\left(1,{ }^{\prime \prime} \mathrm{b}^{\prime \prime}\right)$ & & & \\
\hline \multirow[t]{4}{*}{$2-S$} & & $(2, " a ", I, 3)$ & $(3, " a ", I, 2)$ & $(1,0,1)$ \\
\hline & & & $(3, " b ", 1,2)$ & $(0,1,0)$ \\
\hline & & $\left(2,{ }^{\prime \prime} b^{\prime \prime}, I, 3\right)$ & $(3, " a ", I, 2)$ & $(0,1,0)$ \\
\hline & & & $\left(3, " b^{\prime \prime}, 1,2\right)$ & $(0,1,0)$ \\
\hline
\end{tabular}

Fig. 6. Imperfect Information, three players - Code form

\subsection{Conclusion}

For this game it is not presented the representation in normal form since it points the whole attention in the available strategies for each player so disregarding game whole additional information.

Analysing the representation in code form and the representation in extensive form, it may be concluded that the first presents more information about the game because the extensive form, in the way it is constructed, does not emphasize that the moves referring to players 2 and 3 are simultaneous.

\section{IMPERFECT INFORMATION}

From the presentation of the code form it follows that it specifies:

- The number of players, $\mathrm{N}$, that play the game,

- Each move and if it is sequential or simultaneous,

- Which player moves and which action it makes at each decision point, 
- Which are the available strategies for each player,

- What each player knows about the other players overruling in each decision point,

- The payoffs vector associated to each player's strategies. subgames.

A strategy is a complete action plan in a given game. The solution of a game in code form is perfect in

\section{CONCLUSION}

The whole games representations, approached in the former section, have advantages. The normal form simplifies the game from the mathematical point of view and codifies the extensive form and code form whole information in a matrix. But it is easier to make the game verbal description when it is represented either in the extensive form or in the code form. These forms are also easier to use when it is intended to study part of a game or smaller games. In fact, when facing a very complicate game, one of the methodologies to analyse it is to consider subgames and, in this case, is better to have it represented either in the extensive form or in the code form.

But, having in mind that the importance of a representation lies in the easiness of the gamer interpretation, in the easiness of finding the game solution, in the faithfully of the game information, the code form representation is the one that fulfils these requests.

\section{REFERENCES}

1. G. Asheim, M. Dufwenberg (2000). Deductive reasoning in extensive games. Stockolm University, Department of Economics in its series Research Papers in Economics, $n^{\circ} 2000: 7$.

2. D. Balkenborg (1998). An experiment on forward-versus backwrad induction.Bonn Graduate School of Economics. RePec:bom:bonsfb:268; University of Bonn; June.

3. Games and Information. Basil Blackwell Oxford, 1989.

4. T. Ferguson. Game Theory. 2000.

5. M.A.M Ferreira (1986). Aplicação dos teoremas de separação na programação convexa em espaços de Hilbert. Revista de Gestão, I (2), 41-44.

6. M.A.M. Ferreira, M. Andrade, M. C. Matos (2010). Separation theorems in Hilbert spaces convex programming. Journal of Mathematics and Technology, 1 (5), 20-27.

7. M.A.M. Ferreira, M. Andrade, (2011). Management optimization problems. International Journal of Academic Research, Vol. 3 (2, Part III), 647-654.

8. M.A.M. Ferreira, M. Andrade (2011a). Hahn-Banach theorem for normed spaces. International Journal of Academic Research, 3 (4, Part I), 13-16.

9. M.A.M. Ferreira, M. Andrade (2011b). Riesz representation theorem in Hilbert spaces separation theorems. International Journal of Academic Research, 3 (6, Part II), 302-304.

10. M.A.M. Ferreira M. Andrade (2012). Separation of a vector space convex parts. International Journal of Academic Research, 4 (2), 5-8.

11. M.A.M. Ferreira, M. Andrade, J. A. Filipe (2012). Kuhn-Tucker's theorem for inequalities in infinite dimension. Journal of Mathematics and Technology, 3 (1), 57-60.

12. M.A.M. Ferreira, M. Andrade, M. C. Matos, J. A. Filipe, M. Coelho (2012). Minimax theorem and Nash equilibrium. International Journal of Latest Trends in Finance \& Economic Sciences, 2(1), 3640, 2012.

13. D. Fudenberg, J. Tirole. Game Theory. Cambridge, Mass., MIT Press, 1991.

14. R. Gardner. Juegos para empresarios e economistas. Spanish tranlation by Paloma Calvo and Xavier Vilà; Antoni Bosch, Editor, S. A.; Barcelona, March 1996.

15. R. Gibbons. Un primer curso de teoria de juegos. Spanish translation by Paloma Calvo and Xavier Vilà; Antoni Bosch, Editor, S. A.; Barcelona; October 1993.

16. M.C. Matos, M.A.M. Ferreira (2006). Game representation-Code form. Lecture Notes in Economics and Mathematical Systems, 567, 321-334.

17. M.C. Matos, M.A.M. Ferreira, M. Andrade (2010). Code form game. International Journal of Academic Research, 2(1), 135-141.

18. M.C. Matos, M.A.M. Ferreira, J. A. Filipe, M. Coelho (2010). Prisonner's dilemma: cooperation or treason?. PJQM-Portuguese Journal of Quantitative Methods, 1(1), 43-52.

19. J. Nash (1951). Non-cooperative games. Annals of Mathematics, 54.

20. J. von Neumann, O. Morgenstern. Theory of games and economic behavior. Princeton University Press, Princeton, New Jersey, 1947.

21. J. von Neumann, O. Morgenstern. Theory of games and economic behavior. John Wiley \& Sons Inc., New York, 1967.

22. M.Yildiz. Game theory lecture notes. http://web.mit.edu/14.12/www/02F_lecture102.pdf. Accessed in April 2013. 
Copyright of International Journal of Academic Research is the property of International Journal of Academic Research and its content may not be copied or emailed to multiple sites or posted to a listserv without the copyright holder's express written permission. However, users may print, download, or email articles for individual use. 